\title{
On Some New Linear Generating Relations Involving I-Function of Two Variables
}

\author{
Raghunayak Mishra ,Dr. S. S. Srivastava \\ ${ }^{I}$ Department of Mathematics Narayan Degree College Patti, Pratapgarh (U.P.) \\ ${ }^{2}$ Institute for Excellence in Higher Education Bhopal (M.P.)
}

Abstract: The aim of this research paper is to establish some linear generating relations involving I-function of two variables.

\section{Introduction}

The I-function of two variables introduced by Sharma \& Mishra [2], will be defined and represented as follows:

where

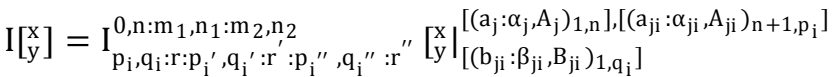

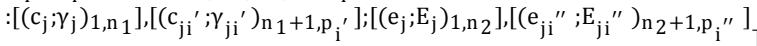

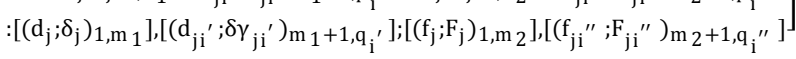

$$
\begin{aligned}
& =\frac{1}{(2 \pi \omega)^{2}} \int_{L_{1}} \int_{L_{2}} \phi_{1}(\xi, \eta) \theta_{2}(\xi) \theta_{3}(\eta) x^{\xi} y^{\eta} d \xi d \eta,
\end{aligned}
$$

$$
\begin{aligned}
& \phi_{1}(\xi, \eta)=\frac{\prod_{j=1}^{n} \Gamma\left(1-a_{j}+\alpha_{j} \xi+A_{j} \eta\right)}{\sum_{i=1}^{r}\left[\prod_{j=n+1}^{p_{i}} \Gamma\left(a_{j i}-\alpha_{j i} \xi-A_{j i} \eta\right) \prod_{j=1}^{q_{i}} \Gamma\left(1-b_{j i}+\beta_{j i} \xi+B_{j i} \eta\right)^{\prime}\right.} \\
& \theta_{2}(\xi)=\frac{\prod_{j=1}^{\mathrm{m} 1} \Gamma\left(\mathrm{d}_{\mathrm{j}}-\delta_{\mathrm{j}} \xi\right) \prod_{\mathrm{j}=1}^{\mathrm{n} 1} \Gamma\left(1-\mathrm{c}_{\mathrm{j}}+\gamma_{\mathrm{j}} \xi\right)}{\sum_{\mathrm{i}^{\prime}=1}^{\mathrm{r}^{\prime}}\left[\prod_{\mathrm{j}=\mathrm{m}_{1}+1}^{\mathrm{q}_{\mathrm{i}}{ }^{\prime}} \Gamma\left(1-\mathrm{d}_{\mathrm{ji}}{ }^{\prime}+\delta_{\mathrm{ji}}{ }^{\prime} \xi\right) \prod_{\mathrm{j}=\mathrm{n}_{1}+1}^{\mathrm{p}^{\prime}} \Gamma\left(\mathrm{c}_{\mathrm{ji}}{ }^{\prime}-\gamma_{\mathrm{ji}}{ }^{\prime} \xi\right)^{\prime}\right.}, \\
& \theta_{3}(\eta)=\frac{\prod_{j=1}^{\mathrm{m} 2} \Gamma\left(\mathrm{f}_{\mathrm{j}}-\mathrm{F}_{\mathrm{j}} \eta\right) \prod_{\mathrm{j}=1}^{\mathrm{n} 2} \Gamma\left(1-\mathrm{e}_{\mathrm{j}}+\mathrm{E}_{\mathrm{j}} \eta\right)}{\sum_{\mathrm{i}}^{\mathrm{r}^{\prime \prime}=1} \prod_{j=\mathrm{m}_{2}+1}^{\mathrm{q}_{\mathrm{i}}^{\prime \prime}} \Gamma\left(1-\mathrm{f}_{\mathrm{ji}}{ }^{\prime \prime}+\mathrm{F}_{\mathrm{ji}}{ }^{\prime \prime} \eta\right) \prod_{j=n_{2}+1}^{\mathrm{p}_{\mathrm{i}}{ }^{\prime \prime}} \Gamma\left(\mathrm{e}_{\mathrm{ji}}{ }^{\prime \prime}-\mathrm{E}_{\mathrm{ji}}{ }^{\prime \prime} \eta\right)^{\prime}},
\end{aligned}
$$

$x$ and $y$ are not equal to zero, and an empty product is interpreted as unity $p_{i}, p_{i^{\prime}}, p_{i^{\prime \prime}}, q_{i}, q_{i^{\prime}}, q_{i^{\prime \prime}}, n, n_{1}, n_{2}, n_{j}$ and $m_{k}$ are non negative integers such that $p_{i} \geq n \geq 0, p_{i^{\prime}} \geq n_{1} \geq 0, p_{i^{\prime \prime}} \geq n_{2} \geq 0, q_{i}>0, q_{i^{\prime}} \geq 0, q_{i^{\prime \prime}} \geq 0,\left(i=1, \ldots, r ; i^{\prime}\right.$ $\left.=1, \ldots, \mathrm{r}^{\prime} ; \mathrm{i}^{\prime \prime}=1, \ldots, \mathrm{r}^{\prime \prime} ; \mathrm{k}=1,2\right)$ also all the A's, $\alpha$ 's, B's, $\beta$ 's, $\gamma^{\prime} \mathbf{s}, \delta$ 's, E's and F's are assumed to be positive quantities for standardization purpose; the definition of I-function of two variables given above will however, have a meaning even if some of these quantities are zero. The contour $L_{1}$ is in the $\xi$-plane and runs from $-\omega \infty$ to $+\omega \infty$, with loops, if necessary, to ensure that the poles of $\Gamma\left(d_{j}-\delta_{j} \xi\right)\left(j=1, \ldots \ldots \ldots . ., m_{1}\right)$ lie to the right, and the poles of $\Gamma\left(1-c_{j}+\gamma_{j} \xi\right)\left(j=1, \ldots, n_{1}\right), \Gamma\left(1-a_{j}+\alpha_{j} \xi+A_{j} \eta\right)(j=1, \ldots, n)$ to the left of the contour.

The contour $L_{2}$ is in the $\eta$-plane and runs from $-\omega \infty$ to $+\omega \infty$, with loops, if necessary, to ensure that the poles of $\Gamma\left(f_{j}-F_{j} \eta\right) \quad\left(j=1, \ldots . ., n_{2}\right)$ lie to the right, and the poles of $\Gamma\left(1-e_{j}+E_{j} \eta\right)\left(j=1, \ldots, m_{2}\right), \Gamma\left(1-a_{j}+\right.$ $\left.\alpha_{j} \xi+A_{j} \eta\right)(j=1, \ldots, n)$ to the left of the contour. Also

$$
\begin{gathered}
R^{\prime}=\sum_{\mathrm{j}=1}^{\mathrm{p}_{\mathrm{i}}} \alpha_{\mathrm{ji}}+\sum_{\mathrm{j}=1}^{\mathrm{p}_{\mathrm{i}}{ }^{\prime}} \gamma_{\mathrm{ji}}{ }^{\prime}-\sum_{\mathrm{j}=1}^{\mathrm{q}_{\mathrm{i}}} \beta_{\mathrm{ji}}-\sum_{\mathrm{j}=1}^{\mathrm{q}_{\mathrm{i}}{ }^{\prime}} \delta_{\mathrm{ji}}{ }^{\prime}<0, \\
S^{\prime}=\sum_{\mathrm{j}=1}^{\mathrm{p}_{\mathrm{i}}} \mathrm{A}_{\mathrm{ji}}+\sum_{\mathrm{j}=1}^{\mathrm{p}_{\mathrm{i}}{ }^{\prime \prime}} \mathrm{E}_{\mathrm{ji}}{ }^{\prime \prime}-\sum_{\mathrm{j}=1}^{\mathrm{q}_{\mathrm{i}}} \mathrm{B}_{\mathrm{ji}}-\sum_{\mathrm{j}=1}^{\mathrm{q}_{\mathrm{i}}{ }^{\prime \prime}} \mathrm{F} \delta_{\mathrm{ji}}{ }^{\prime}<0, \\
U=\sum_{\mathrm{j}=\mathrm{n}+1}^{\mathrm{p}_{\mathrm{i}}} \alpha_{\mathrm{ji}}-\sum_{\mathrm{j}=1}^{\mathrm{q}_{\mathrm{i}}} \beta_{\mathrm{ji}}+\sum_{\mathrm{j}=1}^{\mathrm{m}_{1}} \delta_{\mathrm{j}}-\sum_{\mathrm{j}=\mathrm{m}_{1}+1}^{\mathrm{q}^{\prime}{ }_{\mathrm{ji}}}+\sum_{\mathrm{j}=1}^{\mathrm{n}_{1}} \gamma_{\mathrm{j}}-\sum_{\mathrm{j}=\mathrm{n}_{1}+1}^{\mathrm{p}_{\mathrm{i}}{ }^{\prime}} \gamma_{\mathrm{ji}}{ }^{\prime}>0, \\
V=-\sum_{\mathrm{j}=\mathrm{n}+1}^{\mathrm{p}_{\mathrm{i}}} \mathrm{A}_{\mathrm{ji}}-\sum_{\mathrm{j}=1}^{\mathrm{q}_{\mathrm{i}}} \mathrm{B}_{\mathrm{ji}}-\sum_{\mathrm{j}=1}^{\mathrm{m}_{2}} \mathrm{~F}_{\mathrm{j}}-\sum_{\mathrm{j}=\mathrm{m}_{21}+1}^{\mathrm{q}_{\mathrm{i}}{ }^{\prime}} \mathrm{F}_{\mathrm{ji}}{ }^{\prime \prime}+\sum_{\mathrm{j}=1}^{\mathrm{n}_{2}} \mathrm{E}_{\mathrm{j}}-\sum_{\mathrm{j}=\mathrm{n}_{2}+1}^{\mathrm{p}^{\prime \prime}} \mathrm{E}_{\mathrm{ji}}{ }^{\prime \prime}>0,
\end{gathered}
$$

and $|\arg x|<1 / 2 U \pi,|\arg y|<1 / 2 \bigvee \pi$. 


\section{Linear Generating Relations}

In this section we establish the following linear generating relations:

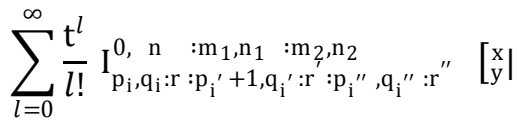

$$
\begin{aligned}
& \ldots, \ldots \ldots \ldots \ldots \ldots,(\lambda-l ; \alpha): \ldots \ldots \ldots, \ldots \ldots \ldots . \\
& =(1+\mathrm{t})^{(\lambda-1)} \mathrm{I}_{\mathrm{p}_{\mathrm{i}}, \mathrm{q}_{\mathrm{i}}: \mathrm{r}: \mathrm{p}_{\mathrm{i}}{ }^{\prime}+1, \mathrm{n}_{\mathrm{i}}{ }^{\prime}: \mathrm{r}^{\prime}: \mathrm{p}_{\mathrm{i}}{ }^{\prime \prime}, \mathrm{q}_{\mathrm{i}}{ }^{\prime \prime}: \mathrm{r}^{\prime \prime}} \quad\left[\begin{array}{r}
\mathrm{x}(1+t)^{\alpha} \\
\mathrm{y}
\end{array}\right.
\end{aligned}
$$

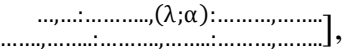

$|\arg x|<1 / 2 U \pi,|\arg y|<1 / 2 \bigvee \pi$, where $U$ and $V$ is given in (2) and (3) respectively;

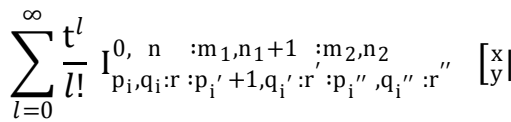

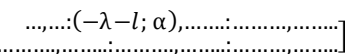

$$
\begin{aligned}
& =(1-\mathrm{t})^{-(\lambda+1)} \mathrm{I}_{\mathrm{p}_{\mathrm{i}}, \mathrm{q}_{\mathrm{i}}: \mathrm{r}: \mathrm{p}_{\mathrm{i}}{ }^{\prime}+1, \mathrm{q}_{\mathrm{i}}{ }^{\prime}: \mathrm{r}^{\prime}: \mathrm{p}_{\mathrm{i}}{ }^{\prime \prime}, \mathrm{q}_{\mathrm{i}}{ }^{\prime \prime}: \mathrm{r}^{\prime \prime}} \quad\left[\begin{array}{r}
\mathrm{x}(1-t)^{-\alpha} \\
\mathrm{y}
\end{array}\right.
\end{aligned}
$$

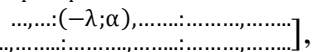

$|\arg x|<1 / 2 U \pi,|\arg y|<1 / 2 V \pi$, where $U$ and $V$ is given in (2) and (3) respectively.

Proof:

To prove (4), consider

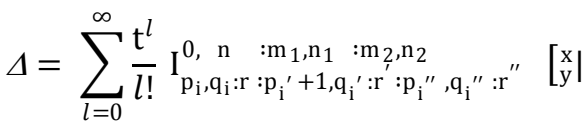

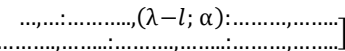

On expressing I-function in contour integral form as given in (1), we get

$$
\begin{aligned}
& \Delta=\sum_{l=0}^{\infty} \frac{\mathrm{t}^{l}}{l !} {\left[\frac{1}{(2 \pi \omega)^{2}} \int_{\mathrm{L}_{1}} \int_{\mathrm{L}_{2}} \phi_{1}(\xi, \eta) \theta_{2}(\xi) \theta_{3}(\eta)\right.} \\
&\left.\times \frac{1}{\Gamma\{\lambda-l-\alpha \xi\}} \mathrm{x}^{\xi} \mathrm{y}^{\eta} \mathrm{d} \xi \mathrm{d} \eta\right] \\
&=\sum_{l=0}^{\infty} \frac{(-\mathrm{t})^{l}}{l !}\left[\frac{1}{(2 \pi \omega)^{2}} \int_{\mathrm{L}_{1}} \int_{\mathrm{L}_{2}} \phi_{1}(\xi, \eta) \theta_{2}(\xi) \theta_{3}(\eta)\right. \\
&\left.\times \frac{\{1-\lambda-\alpha \xi \xi l}{\Gamma\{\lambda-\alpha \xi\}} \mathrm{x}^{\xi} \mathrm{y}^{\eta} \mathrm{d} \xi \mathrm{d} \eta\right] .
\end{aligned}
$$

On changing the order of summation and integration, we have

$$
\begin{array}{r}
\Delta=\frac{1}{(2 \pi \omega)^{2}} \int_{L_{1}} \int_{L_{2}} \phi_{1}(\xi, \eta) \theta_{2}(\xi) \theta_{3}(\eta) x^{\xi} y^{\eta} \\
\times \frac{1}{\Gamma\{\lambda-\alpha \xi\}} \times\left[\sum_{l=0}^{\infty} \frac{(-t)^{l}}{l !}\{1-\lambda-\alpha \xi\}_{l}\right] d \xi d \eta \\
=(1+\mathrm{t})^{\lambda-1} \frac{1}{(2 \pi \omega)^{2}} \int_{L_{1}} \int_{L_{2}} \phi_{1}(\xi, \eta) \theta_{2}(\xi) \theta_{3}(\eta) x^{\xi} y^{\eta} \frac{(1+\mathrm{t})^{-\alpha \xi}}{\Gamma\{\lambda-\alpha \xi\}} d \xi d \eta
\end{array}
$$


which in view of (1), provides (4).

Proceeding on similar lines as above, the results (5) can be derived.

\section{Particular Cases}

I. On specializing the parameters in main formulae, we get following generating relations in terms of I-function of one variable, which are the results given by Khare [1, p.21-23, (2.1) and (2.2)]:

$$
\begin{aligned}
& \left.\sum_{l=0}^{\infty} \frac{\mathrm{t}^{l}}{l !} \mathrm{I}_{\mathrm{p}_{\mathrm{i}}+1, \mathrm{n}, \mathrm{q}_{\mathrm{i}} \mathrm{r}}^{\mathrm{m}}[\mathrm{z} \mid \ldots, \ldots, \ldots-l, \alpha)\right] \\
& =(1+t)^{(\lambda-1)} I_{p_{i}+1, q_{i}: r}^{m, n}\left[z(1+t)^{-\alpha} \mid \ldots, \ldots, \ldots, \ldots, \alpha,\right.
\end{aligned}
$$

provided that $\quad|\operatorname{argz}|<1 / 2 \mathrm{~B} \pi$, where $\mathrm{B}$ is given by $B=\sum_{j=1}^{n} \alpha_{j}-\sum_{j=n+1}^{p_{i}} \alpha_{j i}+\sum_{j=1}^{m} \beta_{j}-\sum_{j=m+1}^{q_{i}} \beta_{j i}>0$;

$$
\begin{aligned}
& \sum_{l=0}^{\infty} \frac{\mathrm{t}^{l}}{l !} \mathrm{I}_{\mathrm{p}_{\mathrm{i}}+1, \mathrm{q}, \mathrm{q}: \mathrm{r}}^{\mathrm{m}, \mathrm{r}+1}\left[\left.\mathrm{z}\right|_{\ldots \ldots,} ^{(-\lambda-l, \alpha), \ldots]}\right. \\
& =(1-t)^{-(1+\lambda)} I_{p_{i}+1, q_{i}: r}^{m, n+1}\left[\left.z(1-t)^{-\alpha}\right|_{\ldots \ldots,} ^{(-\lambda, \alpha), \ldots]}\right] \text {, }
\end{aligned}
$$

provided that $\quad|\operatorname{argz}|<1 / 2 \mathrm{~B} \pi$, where $\mathrm{B}$ is given by $B=\sum_{j=1}^{n} \alpha_{j}-\sum_{j=n+1}^{p_{i}} \alpha_{j i}+\sum_{j=1}^{m} \beta_{j}-\sum_{j=m+1}^{q_{i}} \beta_{j i}>0$.

II. On choosing $\mathrm{r}=1$ in (6) and (7), we get following generating relations in terms of $\mathrm{H}$-function of one variable, which are the results given by Shrivastava \& Shrivastava [3, p.65, (2.1) and (2.2)]:

$$
\begin{aligned}
& \sum_{l=0}^{\infty} \frac{(\mathrm{t})^{l}}{l !} \mathrm{H}_{\mathrm{p}+1, \mathrm{q}}^{\mathrm{m}, \mathrm{n}}\left[\left.\mathrm{z}\right|_{\left(\mathrm{a}_{j}, \alpha_{\mathrm{j}}\right)_{1, \mathrm{p}},(\lambda-l, \alpha)} ^{\left(\mathrm{b}_{\mathrm{j}}, \beta_{\mathrm{j}}\right)_{1, \mathrm{q}}}\right] \\
& \quad=(1+\mathrm{t})^{(\lambda-1)} \mathrm{H}_{\mathrm{p}+1, \mathrm{q}}^{\mathrm{m}, \mathrm{n}}\left[\left.\mathrm{z}(1+\mathrm{t})^{-\alpha}\right|_{\left.\left(\mathrm{a}_{\mathrm{j}}, \alpha_{\mathrm{j}}\right)_{1, \mathrm{p}},\left(\lambda, \beta_{\mathrm{j}}\right)_{1, \mathrm{q}}\right]} ^{(\lambda)}\right],
\end{aligned}
$$

provided that $\quad|\operatorname{argz}|<1 / 2 \mathrm{~A} \pi$, where $\mathrm{A}$ is given by $A=\sum_{j=1}^{n} \alpha_{j}-\sum_{j=n+1}^{p} \alpha_{j}+\sum_{j=1}^{m} \beta_{j}-\sum_{j=m+1}^{q} \beta_{j}>0$;

$$
\begin{aligned}
& \sum_{l=0}^{\infty} \frac{(\mathrm{t})^{l}}{l !} \mathrm{H}_{\mathrm{p}+1, \mathrm{q}}^{\mathrm{m}, \mathrm{n}+1}\left[\left.\mathrm{z}\right|_{\left(\mathrm{b}_{\mathrm{j}}, \mathrm{\beta}_{\mathrm{j}}\right)_{1, \mathrm{q}}} ^{(-\lambda-l, \alpha)\left(\mathrm{a}_{\mathrm{j}}, \alpha_{\mathrm{j}}\right)_{1, \mathrm{p}}}\right] \\
& =(1-t)^{-(1+\lambda)} H_{p+1, q}^{m, n+1}\left[\left.z(1-t)^{-\alpha}\right|_{\left(b_{j}, \beta_{j}\right)_{1, q}} ^{(-\lambda, \alpha),\left(a_{a}, \alpha_{j}\right)_{1, p}}\right],
\end{aligned}
$$

provided that $\quad|\operatorname{argz}|<1 / 2 \mathrm{~A} \pi$, where A is given by $A=\sum_{j=1}^{n} \alpha_{j}-\sum_{j=n+1}^{p} \alpha_{j}+\sum_{j=1}^{m} \beta_{j}-\sum_{j=m+1}^{q} \beta_{j}>0$.

\section{References}

[1]. Khare, Sandeep: An Advanced Study of I-Function of one Variable with Some Applications, Ph.D. Thesis, A.P.S. University, Rewa (M.P.), 2009.

[2]. Sharma C. K. and Mishra, P. L.: On the I-function of two variables and its certain properties, ACI, 17 (1991), 1-4.

[3]. Shrivastava, Shweta and Shrivastava, B. M. L.: Some new generating relations and identities for H-function, Vijnana Parishad Anusandhan Patrika, Vol. 49, No.1, January, 2006, p. 63-77. 